\section{P2-402 TB DIAGNOSTIC INTERVENTIONS IN RESOURCE POOR AREAS: THE ROLE OF SPUTUM FIXERS IN TANZANIA}

doi:10.1136/jech.2011.142976l.32

C Makafu,* Z Mkomwa, M Makame, F Numvire, G Munuo, M Tungaraza, J Mugyabuso, E Bavu, I Makame. PATH, Tanzania

Introduction $\mathrm{TB}$ is among of the major public health problem in Tanzania. The National TB and leprosy program among of their strategy is intensive case finding which has drawbacks taking the fact that Tanzania is a developing country. The coverage of diagnostic centers is very low and some areas are located very interior that it takes hours to reach the diagnostic centers. PATH Tanzania decided to introduce a cadre called sputum fixers; these are community owned resource persons. They screen the community looking for TB suspects. These TB suspects are those with complain of cough. They fix their sputum on slides and send them to the diagnostic centers. These sputum fixers are given bicycles to transport these slides to the laboratories for analysis.

Methods Ten sputum fixers were identified in five districts in Tanzania whereby the sputum of suspects were fixed and sent to laboratory for analysis. Two sputum specimens were taken to the laboratory from each suspect. Any sputum positive of the two slides taken was regarded TB patients.

Results Out of 1195 suspects whose sputum were taken to laboratory for analysis $137(8.7 \%)$ were AFB sputum positive.

Conclusion Introduction of community owned resource persons (sputum fixers) helps in case identification and increase case detection and hence reducing incidence rate after proper treatment.

\section{P2-403 DETERMINANTS OF BED NET USAGE IN CHILDREN UNDER 5 AND HOUSEHOLD BED NET OWNERSHIP IN BIOKO ISLAND, EQUATORIAL GUINEA}

doi:10.1136/jech.2011.142976l.33

\begin{abstract}
${ }^{1} \mathrm{~A} L$ García-Basteiro, ${ }^{*} \mathrm{C}$ Schwabe, ${ }^{2} \mathrm{C}$ Aragon, ${ }^{2} \mathrm{G}$ Baltazar, ${ }^{3} \mathrm{~A}$ M Rehman, ${ }^{4} \mathrm{~A}$ Matias, ${ }^{5} \mathrm{G} N$ Nchama, ${ }^{3} \mathrm{I}$ Kleinschmidt. ${ }^{1}$ Preventative Medicine and Epidemiology Unit, Hospital Clinic, Barcelona, Spain; ${ }^{2}$ Medical Care Development International, Silver Spring, Maryland, USA; ${ }^{3}$ MRC Tropical Epidemiology Group, London School of Hygiene and Tropical Medicine, London, UK; ${ }^{4}$ Medical Care Development International, Malabo, Equatorial Guinea; ${ }^{5}$ Ministry of Health and Social Welfare, Malabo, Equatorial Guinea
\end{abstract}

Background As part of the strategies the Bioko Island Malaria Control Project has implemented in recent years, around 80000 insecticide treated nets (ITN) were delivered to the population in 2007. This study assesses the determinants of bed net usage and bed net ownership in children under 5 .

Methods Data were selected from 2008 and 2009 annual surveys of households who had at least one child under 5. Outcome variables were: sleeping under a net/ITN the night prior to the survey and household ownership of at least one net. The explanatory variables were household characteristics and caregiver's knowledge of malaria. Results A total of 3210 households with 5151 children under 5 were analysed. Higher bed net usage was associated with being sick in the last 14 days prior to the survey, urban area, more years of education of head of households, household ownership of at least one ITN and the year 2009. The proportion of households that owned at least one net in 2009 declined by $32 \%$ when compared to 2008 . Knowing how malaria is prevented and transmitted, having fewer children under 5 and having more children sick in the previous 14 days were associated with higher household net ownership.

Conclusions The fall in bed net usage from 2008 to 2009 is attributable to the striking decline in ownership. Although ownership is similar in rural and urban areas, rural households are less likely to protect their children with nets. Knowledge about malaria seems an important determinant of bed net ownership.
P2-404 HIGH HIV PREVALENCE RATES IN A SEMI-RURAL AREA OF SOUTHERN MOZAMBIQUE: POPULATION-BASED DATA COMPARED WITH ANTENATAL CLINIC PREVALENCE ESTIMATIONS

doi:10.1136/jech.2011.142976l.34

${ }^{1,2} \mathrm{R}$ Gonzalez, ${ }^{2} \mathrm{C}$ Bavo, ${ }^{2} \mathrm{E}$ Pedro, ${ }^{2} \mathrm{~K}$ Munguambe, ${ }^{2} \mathrm{H}$ Boene, ${ }^{2} \mathrm{D}$ Nhalungo, ${ }^{1,2} \mathrm{C}$ Menéndez, ${ }^{1,2} \mathrm{D}$ Naniche. ${ }^{1}$ Barcelona Centre for International Health Research (CRESIB), Barcelona, Spain; ${ }^{2}$ Centro de Investigaçao em Saúde de Manhiça (CISM), Manhiça, Mozambique

Introduction Southern African countries bear an inordinate burden of the global HIV/AIDS pandemic. Monitoring the epidemiology dynamics is critical to identify populations at greatest risk for the infection and assess the maturity of the epidemic.

Methods A cross-sectional population-based study was designed to determine age and sex-specific community HIV prevalence in adults aged 18-47 years old living in Manhiça district, in southern Mozambique. Study candidates were randomly selected from the Demographic Surveillance System in place at the Centro de Investigação em Saúde de Manhiça (CISM) and home-based testing was conducted. The population-based HIV prevalence results were compared with those obtained prospectively from the antenatal clinics (ANC) of Manhiça Health Center.

Results In total, 722 adults participated in the cross-sectional survey. The overall HIV prevalence found in adults aged $18-27$ years was $23.61 \%$ (95\% CI 18.13 to 29.08 ), increasing to $42.62 \%$ (95\% CI 36.39 to 48.85 ) in those aged $28-37$ years and to $45.31 \%$ (95\% CI 39.05 to 51.56$)$ in the $38-47$ years age-group. HIV prevalence estimates were higher in women than in men, especially in younger adults. The overall HIV prevalence found in the study participants was of $37.40 \%$ (95\% CI 33.86 to 40.93 ). Analysis of the ANC data showed an HIV prevalence of $29.35 \%$ (95\% CI 26.70 to 32.00 ) in 952 pregnant women from the same area.

Conclusions Our results show higher HIV prevalence in the population-based survey than in the ANC surveillance system of the same district. This raises issues as to the applicability of ANC prevalence data to estimate overall HIV prevalence, and stresses the need for innovative prevention strategies in sub-Saharan countries.

\section{P2-405 SELF-REPORTED DISCRIMINATION IN EARLY ADOLESCENCE IN A BRAZILIAN BIRTH COHORT: PREVALENCE AND ASSOCIATED FACTORS}

doi:10.1136/jech.2011.142976l.35

${ }^{1,2} \mathrm{D}$ A González, ${ }^{2} \mathrm{H}$ Gonçalves, ${ }^{1} \mathrm{~J}$ L D Bastos, ${ }^{2} \mathrm{~S}$ Dumith. ${ }^{1}$ Universidade Federal de Santa Catarina, Florianópolis, Santa Catarina, Brazil; ${ }^{2}$ Universidade Federal de Pelotas, Pelotas, Rio Grande do Sul, Brazil

Objective To assess the prevalence of and associated factors with self-reported discrimination among adolescents.

Methods Cross-sectional analyses were carried out with data from the Brazilian 1993 Pelotas Birth Cohort Study. Of the 5249 live born children, data on self-reported discrimination, socio-demographic factors and physical attributes were collected on 4452 adolescents interviewed in 2004-2005. Poisson regression was used in crude and adjusted analyses to estimate prevalence ratios (PR).

Results The prevalence of self-reported discrimination attributed to any reason was $16.3 \%$. In adjusted analyses, discrimination was more likely to be reported by girls ( $\mathrm{PR}=1.27,95 \% \mathrm{CI} 1.27$ to 1.48 ); interviewer-classified blacks ( $\mathrm{PR}=1.28,95 \% \mathrm{CI} 1.28$ to 1.57 ); poor respondents ( $\mathrm{PR}=1.58,95 \% \mathrm{CI} 1.23$ to 2.02$)$; those who perceived themselves as very thin or very fat $(\mathrm{RP}=1.81$ and 1.54 , respectively); with family economic problems ( $\mathrm{PR}=1.76,95 \% \mathrm{CI} 1.49$ to 2.08$)$; wearing glasses ( $\mathrm{PR}=1.74,95 \%$ CI 1.45 to 2.10 ); with worse selfperceived dental appearance ( $\mathrm{PR}=1.58,95 \%$ CI 1.21 to 2.07 ); with 